\title{
Normatività e trasgressione nella distribuzione paradigmatica del suppletivismo verbale romanzo
}

\section{Normativity and Transgression in the Paradigmatic Distribution of Verbal Romance Suppletion}

\author{
Fabio Ripamonti [fabioripamonti@yahoo.it] \\ Jihočeská univerzita v Českých Budějovicích, Repubblica Ceca
}

\section{RiASSUNTO}

La struttura apparentemente irregolare dei paradigmi verbali suppletivi nelle lingue romanze può rivelare a un'attenta osservazione una logica ben radicata nel comportamento linguistico dei parlanti che è in grado di conservarsi in modo sostanzialmente uniforme a distanza di tempo e di spazio. Grazie all'introduzione del concetto di 'morfoma' (Aronoff 1994) è possibile valutare queste presunte anomalie come un fenomeno centrale sia nella naturale evoluzione diacronica delle lingue romanze che nella continua riorganizzazione dei paradigmi verbali ad opera dei parlanti. Tuttavia, una delle principali difficoltà di una simile prospettiva risiede nell'impossibilità di astrarre in assoluto i principi generali alla base di queste distribuzioni paradigmatiche. Una simile problematica però può essere adeguatamente affrontata, sebbene non del tutto risolta, se la ricerca su questo tema ricorre alle teorie, sia passate che presenti, relative al concetto di "norma linguistica", all'interno delle quali i morfomi verbali romanzi possono essere interpretati come espressione della libertà di un sistema linguistico.

\section{PAROLe CHiAve}

suppletivismo; verbo romanzo; morfoma; paradigma verbale; norma linguistica

\section{Abstract}

The apparently irregular structure of verbal suppletive paradigms in Romance languages reveals in fact a well-established logic in the speakers' language behaviour that has been preserved with uniformity over time and space. Since the introduction of the concept of "morphome" (Aronoff 1994), it has been possible to assess these so-called anomalies as a central phenomenon both in the natural diachronic evolution and in the continuous reorganization of verbal paradigms realized by the speakers. Nevertheless, one of the main difficulties of such a perspective when applied to Romance languages stands in the impossibility of making absolute abstractions of general principles that are at the base of these distributions in verbal paradigms. However, it is possible to deal appropriately with this kind of problems, albeit not definitely solve them, if the research into this issue refers to theories, past and present, about the concept of "linguistic norm", where Romance verbal morphomes can be described as an expression of the freedom in a language system. 


\section{KEYWORDS}

suppletion; ŕomance verb; morpheme; verbal paradigm; linguistic norm

RICEVUTO 2017-04-20; ACCETTATO 2017-06-26

Il collasso strutturale a cui andò incontro il latino nel periodo post classico, o comunque in quello riconducibile alla ben nota etichetta di "latino volgare", diede avvio a una serie di effetti a catena che portarono le emergenti lingue romanze a strutturarsi secondo modalità spesso sconosciute in precedenza. Ciò si può notare soprattutto nella morfologia del verbo, dove si verificò una complessa riorganizzazione delle forme e delle funzioni attraverso un percorso in cui la forza dei paradigmi ha agito in maniera incisiva. Assumendo un taglio sincronico il risultato può apparire alle volte arbitrario e inspiegabile, ma osservandone lo sviluppo in diacronia in alcuni casi si può invece intravedere l'effetto di movimenti coerenti che hanno portato all'aspetto che i paradigmi verbali presentano tuttora. Tra i diversi fenomeni che solitamente vengono etichettati come "irregolari” nel presente articolo verrà affrontata la questione del suppletivismo verbale romanzo, cercando da un lato di sintetizzare i risultati più recenti della ricerca in corso su questo tema, dall'altro proponendo una descrizione della sua evoluzione in diacronia alla luce di un'interpretazione del concetto di "norma linguistica".

\section{Il dibattito in corso sul fenomeno del suppletivismo}

Tra la fine del XX e il primo decennio del XXI secolo si è assistito a un rinnovato interesse generale per lo studio del suppletivismo, non solo all'interno delle lingue romanze (per una bibliografia generale cfr. Corbett et al., 2005). Tuttavia, più che costituire un campo di ricerca dove è possibile fornire risposte certe ai quesiti che gli studiosi si pongono sulla sua natura e sulla sua origine, il suppletivismo si propone come un ostico banco di prova per la verifica di concetti e di teorie che vanno a interessare la morfologia linguistica più in generale (Mel'čuk 2006: 405; Borjars 2011: 240). Su questo tema, infatti, i vari modelli teorici si sono ritrovati a dover fare i conti con una realtà ben viva nelle lingue naturali della quasi totalità delle famiglie linguistiche in ogni loro epoca storica, ma che mal si confà a una speculazione eccessivamente astratta, alle volte in difficoltà nell'affrontare adeguatamente l'analisi dei fenomeni "devianti".

A seconda delle diverse correnti, l'analisi del suppletivismo è stata nettamente relegata ai margini negli interessi degli studiosi o al contrario è stata considerata essenziale per fornire risposte il più possibile complete ai meccanismi intrinseci dell'evoluzione della morfologia linguistica (Veselinova 2006: 18-22, 27). Il paradigma generativo-trasformazionale, escludendo nelle sue prime formulazioni lo studio della morfologia, ha ovviamente ignorato anche lo studio del suppletivismo, relegandolo alle questioni relative al lessico, e pertanto argomento che non meritava attenzione. Allo stesso modo anche il modello della Morfologia naturale, basandosi su una scala di trasparenza morfotattica ben precisa stabilita su un rapporto biunivoco tra forma e significato, vedeva nel suppletivismo il punto più basso della naturalità morfologica e di con- 
seguenza lo considerava un tema periferico, nonostante alcuni studiosi che si rifanno a questa teoria vi abbiano dedicato parte della loro ricerca (cfr. Dressler 1985).

Chi invece considera il suppletivismo come un fenomeno degno di nota ha sottolineato proprio il suo carattere 'naturale', portando come argomento fondamentale per questa opinione la sua sorprendente diffusione nelle lingue del mondo nonostante l'evidente eccentricità del fenomeno (Bybee 1985: 7; Hippisley et alii, 2004: 387; Mel'cuk 2006: 405). Anche le teorie che si basano sul principio dell'economicità ritengono il suppletivismo un fenomeno perfettamente funzionale all'interno della comunicazione, poiché essendo stoccate nel lessico mentale le forme suppletive sono direttamente accessibili a un parlante senza dover essere ricavate da regole produttive (Fertig 1998: 1070; Maiden 2004b: 227). Allo stesso tempo c’è chi ha sottolineato il fatto che il suppletivismo non sia solamente un residuo di un'evoluzione storica, ma il risultato coerente di processi spesso non più produttivi in diacronia, e quindi opachi da un punto di vista sincronico (Veselinova 2006: 24). Infine all'interno della prospettiva che va sotto la definizione di Canonical typology, Greville Corbett (2007) ha stabilito alcuni criteri con cui è possibile persino fornire una tipologia del suppletivismo come fenomeno che si inserisce "canonicamente" in una delle situazioni in cui non si riscontra una perfetta corrispondenza biunivoca tra forma e significato, principio dimostratosi molto spesso nell'analisi linguistica come una mera utopia. Nell'ambito specifico delle lingue romanze la prospettiva di lavoro più feconda di risultati però riguarda i principi relativi alla cosiddetta Autonomous morphology, avviata come ben noto da Mark Aronoff (1994) e portata avanti in ambito anglosassone soprattutto dal gruppo facente riferimento al Research Centre for Romance Linguistics dell'Università di Oxford ${ }^{1}$.

\section{I fattori di incidenza nell'analisi del suppletivismo verbale}

\subsection{Cause e condizioni}

Una questione perennemente al centro del dibattito sul suppletivismo è il tentativo problematico di comprendere appieno la sua natura e le cause che portano non solo al suo sorgere ma anche alla sua resistenza nel tempo. Già nelle prime formulazioni su questo tema risalenti al pioneristico studio di Osthoff (1899) si ritrova un'osservazione molto semplice, ossia che nelle lingue naturali il suppletivismo coinvolge elementi molto prossimi alla sfera d'azione della vita quotidiana degli esseri umani (Nahbereich) sia nelle scelte lessicali (cfr. it. mucca/toro; ingl. son/daughter) che in quelle morfologiche (cfr. i paradigmi dei verbi più comuni nelle diverse lingue del tipo 'essere, andare, fare...' oppure i comparativi di aggettivi ad alta frequenza: ingl. good - better - best, ceco dobrý - lepší - nejlepší). Ovviamente questo concetto ha ricevuto una critica sistematica a causa della sua evidente inconsistenza scientifica (Fertig 1998: 1071-1074), tuttavia non si configura nemmeno come un principio assolutamente errato, ma necessita solamente di essere inserito in un più ampio contesto che prelude a spiegazioni di tipo psicolinguistico o cognitivo basate ad esempio sul ruolo della deissi spaziale, sull'analisi del discorso o della testualità (Markey 1985: 62).

1 Consultabile al link http://romverbmorph.clp.ox.ac.uk/ [2017-04-19] 
Questa prima questione che ci si pone presenta già a suo modo il generale carattere del dibattito sul suppletivismo, poiché a seconda delle opinioni degli autori si possono trovare diverse definizioni che spesso non concordano tra di loro (Veselinova 2006: 4; Mel’čuk 2006: 405). Il disaccordo ha inizio persino nel momento in cui si devono valutare i confini del suppletivismo. Le opinioni infatti variano da un estremo in cui si potrebbero collocare autori come Markey (1985), Mel'čuk (1994) e Grossmann - Rainer (2004: 17), i quali includono anche i casi di morfologia derivazionale, o altri ancora (Bhat 1967; Myhill 2001) che si spingono fino a inglobare questioni di semantica lessicale in base alle quali anche la sinonimia andrebbe considerata come una forma di suppletivismo, e all'altro estremo autori come Rudes (1980: 660) e Bybee (1985) che restringono la categoria di suppletivismo solo e esclusivamente ai casi in cui sono coinvolti due o più lessemi non legati in alcun modo da relazioni riconducibili a spiegazioni etimologiche o a qualsiasi naturale evoluzione diacronica.

Nonostante le differenze nell'approccio al tema, è unanime la convinzione che il suppletivismo è un fenomeno comprensibile solo assumendo una globale prospettiva diacronica sull'evoluzione dei paradigmi (Rudes 1980: 557, 663; Veselinova 2006: 97; Börjars - Vincent 2011: 241). Oltre a ciò, è necessario osservare che la vicinanza semantica tra i lessemi coinvolti è una condizione pressoché necessaria in una relazione suppletiva (Rudes 1980: 667; Maiden 2004b: 229), sebbene non sia sempre facile stabilirne una tipologia; in questo caso la situazione che si viene a creare solitamente presenta un lessema dal significato più specifico con la funzione di "donatore" di alcune forme verso un secondo lessema "ricevente" dal significato più generale. Tuttavia nel panorama delle lingue romanze esiste almeno un'eccezione a questa prospettiva: nel paradigma del verbo col significato di "dare" il normanno insieme ad alcuni dialetti liguri da una parte e il siciliano dall'altra presentano una distribuzione opposta e complementare tra i lessemi dare e donare nel presente indicativo (Smith 2011: 294-295). La condivisione di tratti morfosintattici tra i lessemi coinvolti è un'altra condizione necessaria affinché si possa parlare di suppletivismo nei paradigmi verbali (Rudes 1980: 667-668), per quanto pure in questo caso esista almeno un'eccezione riscontrabile in antico toscano per il sostantivo uscio, che ha intaccato la coniugazione regolare del verbo escire (Maiden 2004b: 235-238).

\subsection{Irregolarità, difettività, frequenza, analogia}

Altre condizioni di cui spesso si discute nella letteratura di settore paiono invece meno generalizzabili e presentano problematiche più complesse. Solitamente il suppletivismo verbale compare in paradigmi che presentano una forte irregolarità già nei loro precursori (cfr. lat. ire, esse 'andare, essere' oppure ingl. ant. gā/ēode 'andare'), tanto da spingere a stabilire una sorta di equazione tra i due principi. In effetti quando si discute di suppletivismo si fa riferimento a un fenomeno che presenta la massima irregolarità nella morfologia di alcune celle di un paradigma e una prospettiva diacronica molto spesso pare confermare che una precedente irregolarità di un lessema agisce come fattore di facilitazione per il sorgere del suppletivismo (Rudes 1980: 667). Questa evidente considerazione però pone ulteriori questioni che rimangono sostanzialmente inspiegabili. Non è chiaro ad esempio il motivo per cui, nonostante l'irregolarità morfologica, le diverse forme di un lessema conservano senza problemi la regolarità semantica (Mel’čuk 
2006: 415). Se si osserva invece il passaggio dal latino alle lingue romanze non è spiegabile per quale motivo il suppletivismo compaia in verbi precedentemente regolari (ovviamente fatti salvi i casi in cui è possibile fornire una motivazione di tipo fonologico allorigine delle forme suppletive), mentre ad esempio un verbo latino anomalo come dare tenda a livellare le sue forme, perlomeno in alcune delle sue celle, verso un paradigma regolare. Per di più va ricordato che i restanti verbi anomali latini (volle 'volere', esse 'essere'...) hanno comunque sviluppato una struttura paradigmatica diversa da quella originale nelle lingue romanze.

Un'altra condizione che spesso viene chiamata in causa per spiegare il sorgere e il proliferare del suppletivismo è la difettività di un paradigma (Aronoff - Fudeman 2011: 271). Questo fenomeno sarebbe alla base di una strategia volta a completare un paradigma mancante di alcune forme con altre prese in prestito da lessemi diversi, creando in questo modo i presupposti per il suppletivismo. Una simile spiegazione appare evidentemente troppo semplicistica e incompleta (Börjars - Vincent 2011: 244; Hinzelin 2011: 289-290), poiché non sembra possibile immaginare una situazione in cui verbi molto frequenti in una qualsiasi fase dell'evoluzione diacronica siano stati privi di qualche cella del loro paradigma. Tutt'al più la difettività può essere legata al suppletivismo come uno dei fenomeni "non canonici" descritti nell'ambito della Canonical typology (cfr. Baerman - Corbett - Brown 2010; soprattutto gli studi di Boyé - Cabredo Hofherr e di Maiden - O’Neill), intesi come manifestazione di una logica simile e per certi versi complementare.

Ancora più complesso e non definitivamente chiaro è il ruolo svolto dalla frequenza e dall'analogia, che vengono qui discussi contemporaneamente poiché pare che i due principi agiscano nello stesso modo; il suppletivismo infatti compare in genere nei paradigmi a più alta frequenza che per questo motivo sono in grado di resistere in diacronia al naturale livellamento analogico (Veselinova 2006: 97-105), ma tuttavia è stato fatto notare che allo stesso tempo la frequenza di una distribuzione suppletiva può espandersi analogicamente andando ad intaccare altri paradigmi che in una fase precedente non presentavano forme suppletive. Il paradosso in realtà è solo apparente e non rappresenta altro che un'espressione della complessità cognitiva presente nel comportamento linguistico dei parlanti. La frequente ripetizione di una forma si riflette nella capacità della memoria umana di stoccare nel lessico mentale anche le irregolarità morfologiche e di renderle immediatamente disponibili alla comunicazione, ma ciò vale anche per i processi analogici, che intervengono nel momento in cui invece una forma non è presente nel lessico mentale dei parlanti e deve essere ricreata attraverso l'applicazione di regole produttive (Veselinova 2006: 105; Bybee 2007: 5-22).

Nei paradigmi verbali romanzi è possibile individuare alcune situazioni in cui la frequenza e l'analogia hanno agito con un effetto combinato. La presenza di un inserto velare non etimologico in determinate forme del presente indicativo e del congiuntivo in spagnolo e in italiano in verbi come sp. venir (1a) o it. venire (1b) non è spiegabile solo a partire da un regolare cambio fonetico nel passaggio dal lat. class. vĕnĭo > lat. volg. ${ }^{*}$ venjo $>$ it. ant. vegno > it., sp. mod. vengo, visto che le restanti persone in cui l'inserto velare è presente non avrebbero dovuto produrre un cambiamento simile (Maiden 2001: 44-45), ma si può ipotizzare che l'estensione abbia seguito un percorso analogico a partire dalla $1^{\text {a }}$ pers. sg. dovuto probabilmente alla sua frequenza e quindi alla sua "rilevanza lessicale" nel senso in cui lo intende Bybee (1985). L'attrazione verso un morfoma $N$-pattern di un $U / L$-pattern (cfr. par. 3) di verbi come valoir in francese (Maiden 
2004a: 379) (2) poi dimostra come anche un paradigma verbale possa essere sensibile all'attrazione analogica verso una distribuzione altamente frequente:

(1a) Spagnolo moderno venir

\begin{tabular}{|c|c|c|c|c|c|c|}
\hline & $1 \mathrm{sg}$. & 2 sg. & 3sg. & $1 \mathrm{pl}$. & $2 \mathrm{pl}$ & $3 \mathrm{pl}$. \\
\hline $\begin{array}{l}\text { Presente } \\
\text { indicativo }\end{array}$ & $\begin{array}{l}\text { vengo } \\
\text { /'bengo/ }\end{array}$ & $\begin{array}{l}\text { vienes } \\
\text { /'bjenes/ }\end{array}$ & $\begin{array}{l}\text { viene } \\
\text { /'bjene/ }\end{array}$ & $\begin{array}{l}\text { venimos } \\
\text { /be'nimos/ }\end{array}$ & $\begin{array}{l}\text { venís } \\
\text { /be'nis/ }\end{array}$ & $\begin{array}{l}\text { vienen } \\
\text { /'bjenen/ }\end{array}$ \\
\hline $\begin{array}{l}\text { Presente } \\
\text { congiuntivo }\end{array}$ & $\begin{array}{l}\text { venga } \\
\text { /'benga/ }\end{array}$ & $\begin{array}{l}\text { vengas } \\
\text { /'bengas/ }\end{array}$ & $\begin{array}{l}\text { venga } \\
\text { /'benga/ }\end{array}$ & $\begin{array}{l}\text { vengamos } \\
\text { /ben' gamos/ }\end{array}$ & $\begin{array}{l}\text { vengáis } \\
\text { /ben' gajs/ }\end{array}$ & $\begin{array}{l}\text { vengan } \\
\text { /'beygan/ }\end{array}$ \\
\hline
\end{tabular}

\section{Italiano moderno venire}

\begin{tabular}{|c|c|c|c|c|c|c|}
\hline & 1sg. & 2 sg. & 3sg. & $1 \mathrm{pl}$. & 2pl. & 3pl. \\
\hline $\begin{array}{l}\text { Presente } \\
\text { indicativo }\end{array}$ & $\begin{array}{l}\text { vengo } \\
\text { /vengo/ }\end{array}$ & $\begin{array}{l}\text { vieni } \\
\text { /'vjeni// }\end{array}$ & $\begin{array}{l}\text { viene } \\
\text { /'vjene/ }\end{array}$ & $\begin{array}{l}\text { veniamo } \\
\text { /ve'njamo/ }\end{array}$ & $\begin{array}{l}\text { venite } \\
\text { /ve'nite/ }\end{array}$ & $\begin{array}{l}\text { vengono } \\
\text { /'vengono/ }\end{array}$ \\
\hline $\begin{array}{l}\text { Presente } \\
\text { congiuntivo }\end{array}$ & $\begin{array}{l}\text { venga } \\
\text { /'venga/ }\end{array}$ & $\begin{array}{l}\text { venga } \\
\text { /'venga/ }\end{array}$ & $\begin{array}{l}\text { venga } \\
\text { /'venga/ }\end{array}$ & $\begin{array}{l}\text { veniamo / } \\
\text { ve'njamo/ }\end{array}$ & $\begin{array}{l}\text { veniate / } \\
\text { ve'njate/ }\end{array}$ & $\begin{array}{l}\text { vengano / } \\
\text { 'vengano/ }\end{array}$ \\
\hline
\end{tabular}

(2a) Antico francese valoir 'valere'

\begin{tabular}{|c|c|c|c|c|c|c|}
\hline & $1 \mathrm{sg}$. & $2 \mathrm{sg}$. & 3 sg. & $1 \mathrm{pl}$. & $2 \mathrm{pl}$ & $3 \mathrm{pl}$ \\
\hline $\begin{array}{l}\text { Presente } \\
\text { indicativo }\end{array}$ & $\begin{array}{l}\text { vail } \\
\text { /vaא/ }\end{array}$ & $\begin{array}{l}\text { vaus } \\
\text { /vos/ }\end{array}$ & $\begin{array}{l}\text { vaut } \\
\text { /vot/ }\end{array}$ & $\begin{array}{l}\text { valons } \\
\text { /va'lons/ }\end{array}$ & $\begin{array}{c}\text { valez } \\
\text { /va'lets/ }\end{array}$ & $\begin{array}{c}\text { valent } \\
\text { /va'lent/ }\end{array}$ \\
\hline $\begin{array}{l}\text { Presente } \\
\text { congiuntivo }\end{array}$ & $\begin{array}{l}\text { vaille } \\
\text { /'vaאə/ }\end{array}$ & $\begin{array}{l}\text { vailles } \\
\text { /'vaKes/ }\end{array}$ & $\begin{array}{l}\text { vaille } \\
\text { /'vaאə/ }\end{array}$ & $\begin{array}{l}\text { vaillens } \\
\text { /va'Kens/ }\end{array}$ & $\begin{array}{l}\text { vaillez } \\
\text { /va'Kets/ }\end{array}$ & $\begin{array}{l}\text { vaillent } \\
\text { /'vaKent/ }\end{array}$ \\
\hline
\end{tabular}

(2b) Francese moderno valoir 'valere'

\begin{tabular}{|c|c|c|c|c|c|c|}
\hline & 1sg. & 2 sg. & 3sg. & 1pl. & $2 \mathrm{pl}$ & $3 \mathrm{pl}$ \\
\hline $\begin{array}{l}\text { Presente } \\
\text { indicativo }\end{array}$ & $\begin{array}{l}\text { vaux } \\
\text { /vo/ }\end{array}$ & $\begin{array}{l}\text { vaux } \\
\text { /vo/ }\end{array}$ & $\begin{array}{l}\text { vaut } \\
\text { /vo/ }\end{array}$ & $\begin{array}{l}\text { valons } \\
\text { /va'lã/ }\end{array}$ & $\begin{array}{l}\text { valez } \\
\text { /va'le/ }\end{array}$ & $\begin{array}{l}\text { valent } \\
\text { /val/ }\end{array}$ \\
\hline $\begin{array}{l}\text { Presente } \\
\text { congiuntivo }\end{array}$ & $\begin{array}{l}\text { vaille } \\
\text { /vaj/ }\end{array}$ & $\begin{array}{l}\text { vailles } \\
\text { /vaj/ }\end{array}$ & $\begin{array}{l}\text { vaille } \\
\text { /vaj/ }\end{array}$ & $\begin{array}{l}\text { valions } \\
\text { /va'ljã/ }\end{array}$ & $\begin{array}{l}\text { valiez } \\
\text { /va'lje/ }\end{array}$ & $\begin{array}{c}\text { vaillent } \\
\text { /vaj/ }\end{array}$ \\
\hline
\end{tabular}

L'effetto dell'analogia si può spingere anche oltre fino a estendersi a casi anomali, come le forme del verbo ir 'andare' nell'imperfetto indicativo del galiziano nella variante di Finisterre, inspiegabilmente influenzate da quelle di vir 'venire' (3), fenomeno parallelo a quanto avvenuto in alcune varietà di romancio limitatamente alla $1^{\mathrm{a}} \mathrm{sg}$. del presente indicativo tra lo stesso verbo ir e gnir 'venire' (4) (Juge 2013: 181-186). Questi ultimi esempi mostrano che il suppletivismo può essere addirittura insensibile alla natura del cosiddetto "centro deittico" nei verbi di movimento e sembrano porre nuove questioni per quanto riguarda il raggio d'influenza dell'analogia all'interno di un paradigma. 
(3) Galiziano ir 'andare'

\begin{tabular}{|c|c|c|c|c|c|c|}
\hline & 1sg. & $2 \mathrm{sg}$. & 3sg. & 1pl. & 2pl. & 3pl. \\
\hline $\begin{array}{l}\text { Imperfetto } \\
\text { indicativo }\end{array}$ & $\begin{array}{l}\text { iña } \\
\text { /'ina/ }\end{array}$ & $\begin{array}{l}\text { iñas } \\
\text { /'inas/ }\end{array}$ & $\begin{array}{l}\text { iña } \\
\text { /'ina/ }\end{array}$ & $\begin{array}{l}\text { iñamos } \\
\text { /'inamos/ }\end{array}$ & $\begin{array}{l}\text { iñades } \\
\text { /'inaðes/ }\end{array}$ & $\begin{array}{l}\text { iñan } \\
\text { /'inan/ }\end{array}$ \\
\hline
\end{tabular}

Galiziano vir 'venire'

\begin{tabular}{|l|c|c|c|c|c|c|}
\hline & 1 sg. & 2sg. & 3sg. & $1 \mathrm{pl}$. & 2 pl. & 3 pl. \\
\hline $\begin{array}{l}\text { Imperfetto } \\
\text { indicativo }\end{array}$ & $\begin{array}{c}\text { viña } \\
\text { /'bina/ }\end{array}$ & $\begin{array}{c}\text { viñas } \\
\text { /'binas/ }\end{array}$ & $\begin{array}{c}\text { viña } \\
\text { /'bina/ }\end{array}$ & $\begin{array}{c}\text { viñamos / } \\
\text { 'binamos/ }\end{array}$ & $\begin{array}{c}\text { viñades / } \\
\text { 'binaðes/ }\end{array}$ & $\begin{array}{c}\text { viñan } \\
\text { /'binan/ }\end{array}$ \\
\hline
\end{tabular}

(4) Romancio (surmirano) ir 'andare'

\begin{tabular}{|c|c|c|c|c|c|c|}
\hline & $1 \mathrm{sg}$. & $2 \mathrm{sg}$. & $3 \mathrm{sg}$. & 1pl. & 2pl. & 3pl. \\
\hline $\begin{array}{l}\text { Presente } \\
\text { indicativo }\end{array}$ & $\begin{array}{l}\text { vegn } \\
\text { /'vej/ }\end{array}$ & $\begin{array}{l}\text { vest } \\
\text { /'veft/ }\end{array}$ & $\begin{array}{l}\text { vo } \\
\text { /'vo/ }\end{array}$ & $\begin{array}{l}\text { dyaindza } \\
\text { /'Jaincza/ } \\
\text { dyagn } \\
\text { /'„aa// }\end{array}$ & $\begin{array}{l}\text { dyais } \\
\text { /'Jais/ }\end{array}$ & $\begin{array}{c}\text { von } \\
\text { /'von/ } \\
\text { vonen } \\
\text { /'vənən/ }\end{array}$ \\
\hline
\end{tabular}

Romancio (surmirano) gnir 'venire'

\begin{tabular}{|c|c|c|c|c|c|c|}
\hline & $1 \mathrm{sg}$. & $2 \mathrm{sg}$. & $3 \mathrm{sg}$. & $1 \mathrm{pl}$. & $2 \mathrm{pl}$ & $3 \mathrm{pl}$. \\
\hline $\begin{array}{l}\text { Presente } \\
\text { indicativo }\end{array}$ & $\begin{array}{l}\text { vegn } \\
\text { /'vep/ }\end{array}$ & $\begin{array}{l}\text { vegnest } \\
\text { /'vejəft/ } \\
\text { vegnas } \\
\text { /'venas/ }\end{array}$ & $\begin{array}{l}\text { vegn } \\
\text { /'ven/ }\end{array}$ & $\begin{array}{l}\text { gnindza } \\
\text { /'nindza/ } \\
\text { gnin } \\
\text { /'nin/ }\end{array}$ & $\begin{array}{l}\text { gnis } \\
\text { /'nis/ }\end{array}$ & $\begin{array}{l}\text { vegnen } \\
\text { /'vejən/ }\end{array}$ \\
\hline
\end{tabular}

Nonostante questi fenomeni appaiano a prima vista immotivati e arbitrari, tuttavia tendono a ricalcare alcuni patterns sostanzialmente identici che sono ben presenti nell'evoluzione diacronica delle lingue romanze, dimostrando quindi di possedere una profonda consistenza psicologica per i parlanti. La natura 'morfologizzante' di queste distribuzioni è stata ampiamente appurata nell'ambito dell'Autonomous Morphology (Maiden et al. 2011; Ledgeway - Maiden 2016), all'interno della quale, grazie al ricorso al concetto di 'morfoma', si è cercato di dar conto di come le presunte anomalie di un lessema in realtà agiscono secondo una logica che si potrebbe definire di tipo 'carsico'.

\section{Le distribuzioni morfomiche dei paradigmi suppletivi nei verbi romanzi}

Negli studi di morfologia verbale romanza, il concetto di 'morfoma', elaborato dal linguista canadese Mark Aronoff (1994), si è dimostrato particolarmente proficuo nell'affrontare con una nuova luce alcuni problemi discussi da tempo (per un contributo recente cfr. Luís - Bermúdez Otero 2016). Per 'morfoma' si intende un'entità morfosintattica astratta che si comporta come una funzione matematica $f(x)$, a cui corrispondono diverse realizzazioni in base alle condizioni poste dalla funzione stessa e che regola la distribuzione delle forme all'interno di un paradigma 
(O’Neill 2011: 70). Il ruolo dei morfomi come modelli cognitivamente pregnanti è stato ampiamente dimostrato non solo nella diacronia delle lingue romanze ma anche attraverso esperimenti compiuti con parlanti nativi (Bybee - Pardo 1981; Giraudo - Montermini - Pirrelli 2010; Nevins - Rodrigues 2012; Nevins - Rodrigues - Tang 2015).

Nel corso del tempo la descrizione di questo concetto si è parzialmente modificata e al momento attuale varia ancora a seconda degli autori, in particolar modo nel caso del verbo romanzo sembra che sia difficoltoso arrivare a una definizione 'in positivo' di cosa sia un morfoma, poiché i motivi che portano i paradigmi verbali a strutturarsi in base a questo template paiono ancora assolutamente arbitrari. Martin Maiden (2007: 510) ha identificato tre caratteristiche essenziali dei morfomi nelle lingue romanze: 1) coerenza interna (quando cambia una cella di un paradigma cambiano anche le altre celle ad essa collegate); 2) convergenza (all'interno di un morfoma vengono assegnati tratti fonologici comuni); 3 ) attrazione (una distribuzione morfomica è in grado di attirare verso di sé anche altri paradigmi). Sempre allo stesso autore poi si devono anche le denominazioni dei patterns morfomici più diffusi nel verbo romanzo (Maiden 2001b: 41-43): U/L pattern ( $1^{\text {a }}$ sg. del pres. ind. e le forme del sg. nel pres. cong., a cui può aggiungersi la $3^{\text {a }}$ pl. del pres. ind., cfr. sp. poner, it. spegnere), $N$-pattern, (le forme del sg. e della $3^{\text {a }} \mathrm{pl}$. del pres. ind. e spesso anche cong., oltre alla $2^{a}$ sg. dell'imper., cfr. fr. devoir, lad. bëri), PYTA roots (acronimo di 'Perfecto Y Tiempos Afines', ossia le forme dei verbi soprattutto iberoromanzi legati tra loro dalla presenza di una stessa base e che interessano il preterito ind., l'imper. cong., oltre alle forme che continuano il piucch. lat. e il fut. ant., cfr. port. fazer, sp. haber).

Nei morfomi descritti qui sopra si possono riscontrare alcune peculiarità. Innanzitutto sono tipicamente romanzi, ossia non sono rintracciabili in nessuna altra famiglia linguistica; in secondo luogo, stanno a dimostrare come, a differenza di altre parti del discorso, la morfologia verbale romanza è andata complicandosi rispetto al latino, dove l'allomorfia delle basi era limitata solo a un piccolo gruppo di verbi anomali, come ad es. ferre o posse, oppure svolgeva una funzione distintiva tra i tempi dell'infectum e del perfectum; analizzandone poi l'evoluzione diacronica, bisogna constatare che una distribuzione morfomica romanza affonda le sue radici in fenomeni di tipo fonologico già presenti in latino (l'accento del presente indicativo della III coniugazione latina corrisponde a un $\mathrm{N}$-pattern e probabilmente ne è l'origine) o dovuti a un regolare processo di cambiamento fonetico romanzo (l'intacco delle consonanti velari davanti a vocale palatale, cfr. lat. legis, it. leggi; oppure l'effetto di uno yod secondario, cfr. lat. venio, it. ant. vegno, port. venho) che però acquisiscono successivamente una pregnanza esclusivamente morfologica, in cui la fonologia ha un ruolo secondario nonostante alcune rare eccezioni (cf. Maiden 2013 per la discussione su alcuni gerundi in rumeno e in italiano).

L'uniformità delle distribuzioni morfomiche però non esaurisce completamente il problema del suppletivismo verbale. Alcuni casi infatti rimangono tuttora opachi: i morfomi misti definibili come blended morphomes, o secondo la terminologia di Martin Maiden (2009: 64) come clash of morphomes, ossia una distribuzione di compromesso tra due morfomi; l'estensione dell'affisso incoativo -sc-nell'imperfetto francese; la compresenza dei lessemi a voi/a vrea entrambi col significato di 'volere', il primo di origine slava, il secondo di origine latina, nello stesso paradigma in rumeno; alcuni casi di allomorfia presenti nei PYTA roots del portoghese, come in fazer 'fare'; le incursioni nel romancio soprasilvano ir 'andare' delle forme fuse di ambulare e vadĕre nel lessema di base meare; il netto contrasto tra le basi del futuro e quelle del 
non futuro in francese. Pertanto lo stesso concetto di 'morfoma', lungi dall'essere un rigido meccanismo di descrizione delle irregolarità paradigmatiche, necessita evidentemente di maggiore malleabilità nella sua applicazione ai reali fenomeni presenti nella morfologia verbale romanza. La stessa elasticità che a nostro avviso andrebbe applicata nell'approccio verso il concetto di norma linguistica, di cui quello di morfoma è a suo modo parte integrante.

\section{Morfomi e norma linguistica}

La sostanziale regolarità con cui si manifestano le distribuzioni morfomiche delle forme suppletive sembra suggerire la necessità di concepire i paradigmi verbali romanzi tenendo conto di un approccio elastico verso il concetto di 'norma linguistica', così come d'altronde emerge anche dalla riflessione teorica su questo tema perlomeno a partire dalla tradizione strutturalista. La distanza tra l'effettiva realtà delle lingue, non solo romanze, e la loro descrizione normativa pare aver infatti definitivamente spostato i criteri di giudizio sulle espressioni linguistiche da una dicotomia "giusto-sbagliato" a una scala di valutazione ai cui estremi vanno collocati i giudizi di "adeguato-non adeguato" in cui in ultima analisi si integrano i diversi livelli di ricerca, dall'analisi diacronica in senso stretto fino alle teorie della comunicazione.

\subsection{Lo strutturalismo classico di fronte alla norma linguistica}

Tralasciando quanto già in Saussure veniva più o meno velatamente espresso a proposito della normatività linguistica soprattutto in relazione alla sua funzione sociale, è però nella tradizione del Circolo linguistico di Praga (CLP) che il tema della norma ha iniziato a occupare un ruolo centrale nella riflessione teorica e questo già a partire dalle famose Thèses del 1929. Benché non vi sia mai stata una presa di posizione comune da parte dei vari membri, è possibile comunque individuare alcuni principi generali che, pur concepiti soprattutto per la norma scritta del ceco, paiono utili anche per l'analisi dei morfomi romanzi. Innanzitutto la norma linguistica non deve coincidere con l'uso, ma nemmeno allontanarsene, poiché tra di loro non esiste una differenza di status, bensì solo di consapevolezza e di obbligatorietà. Ciò che costituisce la 'norma' è implicitamente interno alla lingua stessa, ossia una comunità di parlanti interiorizza in ogni momento ciò che considera normativo per poter intervenire nello stabilire se la norma stessa viene rispettata o meno e quale livello di tolleranza va accettato nei casi della sua trasgressione (Nebeská 1996: 15-18). Un tratto tipico del CLP poi è l’importanza attribuita al principio di 'funzionalitä, che va a completare quello di 'sistema' nella classica accezione strutturalistica. In questo senso la normatività di un elemento si colloca tra la stabilità garantita da una lingua in quanto sistema e la variabilità funzionale attraverso la quale si esprime la libertà dei parlanti. Questi due estremi non possono situarsi a un'eccessiva distanza tra di loro, poiché altrimenti verrebbe meno la base stessa della stessa comunicazione linguistica, ma devono poter realizzare tra di loro una "stabilità elastica", per seguire la celebre definizione di Vilém Mathesius (Nebeská 1996: 24-32). Riassumendo, quindi, per il CLP il concetto di 'norma' si configura come uno spazio di sistematicità fondato su una consapevolezza interiorizzata, conscia o meno che sia, dei 
parlanti, che sono in grado di valutarne attivamente i risultati in base agli obiettivi comunicativi da raggiungere all'interno di un contatto sociale.

Nella ben nota dicotomia saussureana di langue-parole, il linguista rumeno Eugeniu Coseriu, preceduto in un approccio simile anche da Louis Hjelmslev, ha avanzato l'idea di suddividere il primo dei due termini in due ulteriori concetti, proponendo quindi una tripartizione dei livelli di analisi: sistema - norma - parole (1952; 1988 passim). Nella sua accezione, per 'sistema' s'intende l'insieme delle possibilità a disposizione dei parlanti, una sorta di bacino a cui attingere nel momento in cui si deve compiere una scelta linguistica; per 'norma' invece s'intende l'insieme degli obblighi a cui una lingua impone i suoi parlanti. Secondo Coseriu, i due principi sono sempre presenti nel comportamento linguistico: il 'sistema' permette la creatività e la libertà comunicativa, rappresenta la dinamicità della lingua e la potenzialità dei cambiamenti; la 'norma' è costituita dalla tradizione espressa storicamente da una lingua e dalle sue convenzioni, garantisce una base comune di comunicazione tra i parlanti facendo rispettare le regole che un gruppo linguistico si impone. I due principi sono in continua comunicazione tra di loro, la 'norma' attinge all'insieme di possibilità, di variazioni potenziali che le vengono offerte dal 'sistema' e le rende regole concrete e accettate da una comunità di parlanti. Il cambio linguistico avviene attraverso proprio questa dinamica, poiché non vi è mai nella 'norma' ciò che non era già prima nel 'sistema'. Il riferimento alle potenzialità del 'sistema' permette poi di fornire una spiegazione la ragione per cui i parlanti possono comprendere anche ciò con cui non sono mai entrati in contatto precedentemente, ma anche perché le deviazioni da una 'norma' non compromettono la comunicazione se viene salvaguardato il doppio asse di solidarietà che ogni lingua deve presentare, ossia quello verso la tradizione e quello verso l'interlocutore. Comprendere il motivo per cui solo alcune delle innovazioni presenti nel 'sistema' diventino 'norma' richiederebbe la spiegazione della libertà che caratterizza in generale il comportamento linguistico, basata non su cause deterministiche ma sulla finalità espressiva.

Secondo un punto di vista strettamente strutturalistico, dunque, i morfomi potrebbero essere considerati come un'espressione della libertà del sistema linguistico all'interno dei paradigmi verbali, grazie al fatto che i parlanti sono attivamente impegnati, con maggiore o minore consapevolezza, ad analizzare la tipologia di relazione che le diverse celle di un verbo instaurano tra di loro anche nel caso in cui venga introdotto un cambiamento in una di esse.

\subsection{L'approccio della prospettiva semantica}

A cavallo tra gli anni Ottanta del XX secolo e i primi anni del XXI il tema della norma linguistica è stato affrontato in particolar modo da prospettive di tipo semantico-pragmatico che non raramente si sono spinte ad affrontare questo argomento attraverso approcci filosofici. Rispetto a quanto espresso dagli autori strutturalisti, in queste teorie il riferimento all'uso di una comunità linguistica come essenza della norma non si è limitato solo all'aspetto quantitativo, in base al quale un gruppo linguistico è sovrano nel decidere in ogni momento cosa sia giusto o sbagliato nella propria lingua, poiché tutto ciò non arriva comunque a toccare i motivi di fondo che convalidano l'esistenza di una norma e non di un'altra. Tuttavia è un dato incontrovertibile che nello stabilire il valore di una norma l'elemento sociale giochi un ruolo essenziale da cui 
non si può prescindere; il comportamento linguistico infatti non segue le leggi a-storiche della natura o dei sistemi formali, come la matematica o la biologia, ma dipende strettamente dall'intervento dei suoi fruitori. Nel caso della norma linguistica, dunque, non è applicabile la logica di tipo apodittico di stampo aristotelico basata su una dicotomia "vero-falso", perché snatura l'essenza stessa del linguaggio umano (Itkonen 2008: 287; Rastier 2008: 4). Su questo punto pare significativa una riflessione molto semplice ma efficace del filosofo del linguaggio Ludvig Wittgenstein riportata in Itkonen (2008: 280-290) definita come "private-language argument": nel caso del linguaggio umano il celebre principio cartesiano del cogito ergo sum, che presupponeva nell'esistenza a priori dell'io la base della conoscenza, non è da considerarsi sufficiente, poiché è possibile riconoscere l'esistenza e la correttezza di un comportamento linguistico individuale solo all'interno delle interazioni con altri parlanti. Se così non fosse, ogni individuo avrebbe la possibilità di crearsi un linguaggio privato a sé del quale però non sarebbe in grado di valutarne la correttezza proprio perché privo di riscontri intersoggettivi.

Una distinzione terminologica che ha impegnato gli autori è quella che riguarda i termini di "regola" e di "norma". Nel complesso, seppur con diverse sfumature, il primo termine viene solitamente riservato a ciò che in una lingua a un dato momento è considerato stabile, mentre col secondo si è soliti indicarne l'aspetto dinamico, applicando di conseguenza un taglio diacronico si potrebbe affermare che una regola linguistica non è altro che una norma inveterata (Rastier 2008: 5). In questo quadro, dunque, la prospettiva morfomica sui paradigmi verbali romanzi va evidentemente ricondotta allo spazio di competenza della norma, della mutabilità e della negoziazione di una struttura paradigmatica intesa come campo delle possibilità d'espressione morfologica, poiché come già discusso nel par. 3, può accadere che un pattern morfomico agisca con maggiore o minore forza a seconda delle parlate romanze e delle fasi diacroniche a cui si fa riferimento per attirare a sé la struttura di un verbo originariamente estraneo a una distribuzione degli esponenti morfologici.

Riprendendo una distinzione della logica formale, Bartsch (1982: 53-57) sottolinea che in ogni lingua sono presenti più sistemi e più norme formate da tre tipi di regole: 1) "regole di formazione" (da alcuni elementi di base si formano altri elementi; sono regole costruttive, nel senso che definiscono l'essenza di una formula e ne stabiliscono la correttezza); 2) "regole di inferenza" (da una regola se ne deduce un'altra; sono restrittive, limitano l'applicazione di procedure; devono garantire che si possa passare da formule vere a formule vere); 3) "regole strategiche" (gestiscono i primi due tipi di regole per ottenere uno scopo comunicativo). Queste tipologie di regole, che possiedono un diverso valore gerarchico, interagendo tra di loro possono entrare in conflitto e provocare situazioni di instabilità. Come espresso anche nello strutturalismo classico, ovviamente ogni lingua sfrutta questa precaria variabilità per riuscire ad adattarsi alla naturale evoluzione diacronica:

The fact that different systematizations can counteract each other causes the set of systematizations to be unstable. In other words, rules become extended in application, while others become restricted because of this. That is, one systematization can become more general while another may just be distorted by this process. Reanalysis or restructuring of syntactic and morphological structures is a well-known phenomenon in this area, which has been used to explain syntactic and morphological change, [...] This happens because systematization (rule formation) under 
different aspects is a never-ending activity of language learners and language users. In this light the notion of "the system" of a language does not seem very stable nor realistic. (Bartsch 1982: 72-73)

L'accettabilità di una variazione linguistica si basa sulla gerarchia delle norme, in cui in un'ultima analisi la maggiore importanza va delegata alla realizzazione di una comunicazione soddisfacente, anche al di là dell'applicazione dei livelli normativi di rango inferiore. Le deviazioni da una norma, come a volte in alcune teorie di morfologia verbale vengono concepite le celle suppletive dei paradigmi romanzi, non vanno considerate quindi come aberrazioni di un sistema stabile e immutato, ma nostro avviso vanno valutate in base a strategie pragmatiche in cui gioca un ruolo fondamentale da un lato la libertà espressiva dei parlanti e dall'altro il grado di collaborazione e di tolleranza dei riceventi:

$[\ldots]$ to follow the highest norm of communication requires not only following the subjugated (lower) linguistic norms, but also, for the speaker, violating them in a creative way, for the hearer, using the requisite tolerance and cooperation in interpretation. This requisite tolerance in applying semantic norms is the motor of semantic change. Openness and inventiveness in cultural renewal or contact are the fuel that keeps the motor going. The notion of change is inherent in the concept of semantic norm and allows our language to be sufficiently vague and to have the openness of context-dependent meaning, for it to be adjusted as a means for expressing the results of changes in our natural, social, technical, and cultural environment. (Bartsch 1982: 78-79)

Ancora più avanti si spinge Rastier quando afferma che lo spazio della norma è il punto di contatto tra la linguistica della langue e quella della parole di stampo saussureano (Rastier 2008: 5-7) in quanto solo l'analisi delle norme può dar conto dei meccanismi di regolazione tra le due prospettive:

Alors que la linguistique de la langue, conformément à la problématique logico-grammaticale, objective les unités par leur position spatiale, la linguistique de la parole les objective principalement par leur position temporelle, conformément à la problématique rhétorico-herméneutique. [...] Bref, si une langue n'est pas composée d'un et d'un seul système, chacun de ses niveaux de description comporte des normes et des règles qui évoluent selon des temporalités différenciées, en fonction de paramètres internes et externes différents. La langue comme système unique le cède alors à la langue conçue comme articulation et recherche d’optimisation entre des systèmes partiels relativement autonomes et dont les principes structuraux restent compatibles entre eux, mais non systématiquement intercorrélés. Certains sont centraux, au sens où ils sont à l’œuvre dans toutes les manifestations linguistiques (ex. morphologie, syntaxe); d'autres sont facultatifs (réalisations prosodiques, typographie, etc.). Les règles linguistiques concernent les systèmes partiels, mais non leur interaction, qui obéit à des normes plutôt qu’à des règles. (Rastier 2008: 9) 


\section{Conclusioni}

La comprovata esistenza di modelli morfomici nella conformazione dei paradigmi verbali romanzi rende a nostro avviso più articolato e complesso il giudizio su ulteriori questioni di tipo normativo per quanto riguarda la morfologia dei verbi suppletivi. Sebbene sia ancora evidente che allo stato attuale degli studi non pare possibile fornire una spiegazione soddisfacente al comportamento delle loro strutture morfomiche, non è d'altro canto nemmeno eludibile la questione che esistono delle tendenze nella strutturazione delle irregolarità all'interno dei paradigmi verbali. Da quanto qui espresso, si è voluto contribuire a una riflessione sul rapporto tra la morfologia profonda del verbo romanzo e la norma dei suoi esponenti di superficie con l'obiettivo di descriverne la coerenza e lo spirito. Nell'ambito della Canonical Typology (Corbett 2007) è stato dimostrato che il suppletivismo fa da contraltare ad altri fenomeni solitamente definiti irregolari, come la difettività, la deponenza, l'eteroclisi o il sincretismo, rendendo a nostro avviso necessaria una prospettiva ancora più complessa e globale su questo tema.

\section{Riferimenti bibliografici}

Aronoff, M. (1994). Morphology by itself: stems and inflectional classes. Cambridge, Mass.: MIT Press.

Aski, J. M. (1995). Verbal suppletion: an analysis of Italian, French and Spanish 'to go'. Linguistics, 33, 403-432.

Bartsch, R. (1982). The concepts of «rule» and «norm» in linguistics. Lingua, 58, 51-81.

Bhat, D. N. S. (1967). Lexical suppletion in baby talk. Anthropological Linguistics, 9 (5), 33-36.

Börjars K.; \& Vincent, N. (2011). The pre-conditions for suppletion. In G. Tsoulas, A. Galani, \& G. Hicks (Eds.), Morphology and its Interfaces (pp. 239-265). Amsterdam: John Benjamins.

Bybee, J. L. (1985). Morphology: A Study of the Relation between Meaning and Form. Amsterdam: John Benjamins.

Corbett, G. G. et al. (2005). Resources for Suppletion: A Typological Database and a Bibliography, Morphology and Linguistic Typology. In G. Booij, E. Guevara, A. Ralli, S. Sgroi, \& S. Scalise (Eds.), On-line Proceedings of the Fourth Mediterranean Morphology Meeting (MMM4) Catania, 21-23 September 2003 Università degli Studi di Bologna (pp. 35-44). <https://geertbooij.files.wordpress.com/2014/02/mmm4proceedings.pdf>

Corbett, G. G. (2007). Canonical typology, suppletion, and possible words. Language, 83 (1), 9-42.

Dressler, W. (1985). Sur le statut de la suppléance dans la Morphologie Naturelle. Langages, 20 (78), 4156.

Fertig, D. (1998). Suppletion, natural morphology, diagrammaticity. Linguistics, 36 (6), 1065-1091.

Giraudo, H.; Montermini, F.; \& Pirrelli, V. (2010). Processi cognitivi nell'analisi delle classi verbali dell'italiano: un approccio sperimentale. In V. Bambini, I. Ricci, \& P. M. Bertinetto (Eds.), Linguaggio e cervello - Semantica. Atti del XLII Convegno della Società di Linguistica Italiana (Pisa, Scuola Normale Superiore, 25-27 settembre 2008). Roma: Bulzoni. <https://hal-univ-tlse2.archives-ouvertes.fr/hal00986159/document> 
Hinzelin, M.-O. (2011). Syncretism and Suppletion in Gallo-Romanc Verb Paradigms. In M. Maiden et al. (Eds.), Morphological autonomy. Perspectives from Romance Inflectional Morphology. (pp. 287-310). New York: Oxford University Press.

Hippisley, A. et al. (2004). Suppletion: frequency, categories and distribution of stems. Studies in Language, 28 (2), 387-418.

Itkonen, E. (2008). The central role of normativity in language and linguistics. In J. Zlatev (Ed.), The Shared Mind: Perspectives on Intersubjectivity (pp. 279-305). Amsterdam/Philadelphia: John Benjamins Publishing Company.

Juge, M. (2013). Analogy as a source for suppletion. In R. Kikusava, \& L. A. Reid (Eds.), Historical Linguistics 2011. Selected papers from the 20th International Conference on Historical Linguistics, Osaka, 25 - 30 July 2011. (pp. 175-197). Amsterdam: John Benjamins Publishing Company.

Ledgeway, A.; \& Maiden, M. (Eds.) (2016). The Oxford guide to the romance languages. Oxford: Oxford University Press.

Luís, A. R.; \& Bermúdez-Otero, R. (eds.). (2016). The morphome debate. Oxford: Oxford University Press. Oxford: Oxford University Press.

Maiden, M. (2001). Di nuovo sulle alternanze 'velari’ nel verbo italiano e spagnolo. Cuadernos de Filología Italiana, 8, 39-61.

- (2004a). Verso una definizione morfologica delle lingue romanze. La nuova fisionomia morfologica del romanzo. Aemilianense, I, 357-404.

- (2004b). When lexemes become allomorphs - On the genesis of suppletion. Folia linguistica, 38, 227-256.

. (2007). La linguistica romanza alla ricerca dell'arbitrario. In D. Trotter (Ed.), Actes du XXIVe Congrès International de Linguistique et Philologie Romane, Aberystwyth, 2004 (vol. III) (pp. 506-518). Tübingen: Max Niemeyer.

. (2009). From pure phonology to pure morphology the reshaping of the romance verb. In M. Russo (Ed.), Pour une typologie diachronique et synchronique des langues romanes. Recherches linguistiques de Vincennes (pp. 45-82). <http://rlv.revues.org/1765>

. (2011). Morphophonological innovation. In M. Maiden, J. Ch. Smith, \& A. Ledgeway (Eds.), The Cambridge history of the romance languages (Vol. 1-structures) (pp. 216-267). Cambridge: Cambridge University Press.

- (2016). Morphome. In A. Ledgeway, \& M. Maiden (eds.), The Oxford guide to the romance languages (pp. 708-721). Oxford: Oxford University Press.

Melčuk, I. A. (1994). Suppletion: Toward a logical analysis of the concept. Studies in Language, 18 (2), 339-410.

Myhill, J. (2001). Suppletion, lexical meaning, semantic primitives, and translation data. Linguistics, 39, 761-802.

Nebeská, I. (1996). Jazyk, norma, spisovnost. Praha: Univerzita Karlova.

Nevins, A.; \& Rodrigues, C. (2012). Naturalness biases, 'morphomes', and the Romance first person singular. Paper presented at University of Coimbra, Newcastle University, University of York, and University Paris VII. <http://ling.auf.net/lingbuzz/001469>

Nevins, A.; Rodrigues, C.; \& Tang, K. (2015). The rise and fall of the L-shaped morphome: diachronic and experimental studies. Probus, 27 (1), 101-155. 
O'Neill, P. (2011). The Notion of the Morphom. In M. Maiden, et al. (Eds.), Morphological autonomy. Perspectives from Romance Inflectional Morphology (pp. 70-93). Oxford: Oxford University Press.

Osthoff, H. (1899). Vom Suppletivwesen der indogermanischen Sprachen. Heidelberg: Kommissionsverlag von Alfred Wolff.

Rastier, F. (2008). Conditions d'une linguistique des normes. In A. Steuckardt, \& G. Siouffi (Eds.), Les linguistes et la norme - Aspects normatifs du discours linguistique, (pp. 3-20). Berne: Peter Lang.

Rudes, B. A. (1980). On the nature of verbal suppletion. Linguistics, 18, 655-676.

Smith, J. Ch. (2011). Change and continuity in form - function relationships. In M. Maiden, J. Ch. Smith, \& A. Ledgeway (eds.), The Cambridge history of the romance languages (Vol. 1-Structures) (pp. 268-317). Cambridge: Cambridge University Press.

Veselinova, L. N. (2006). Suppletion in Verb Paradigms: Bits and Pieces of the Puzzle. Amsterdam: John Benjamins Publishing Company. 
